(BPD). Neither score predicted necrotizing enterocolitis or spontaneous intestinal perforation (NEC/SIP), or severe retinopathy of prematurity (ROP).

Conclusion The HRC index in the first day after birth predicts death and multiple morbidities in ELBW infants and is strongly correlated with SNAP-II, an established illness severity score.

\section{PS-047 TRADEOFFS BETWEEN MORTALITY AND MORBIDITY FOR VERY PRETERM INFANTS: RESULTS FROM THE EPICE COHORT}

J Zeitlin', AK Edstedt Bonamy², M Bonet', ES Draper ${ }^{3}$, E Epice Research Group ${ }^{4}$. 'Obstetrical Perinatal and Pediatric Epidemiology Research Team Center for Epidemiology and Biostatistics (U1153), Inserm, Paris, France; 'Department of Women's and Children's Health, Karolinska Institutet, Stockholm, Sweden; ${ }^{3}$ Department of Health Science, University of Leicester, Leicester, UK; ${ }^{4}$ Group Author, European Project, Paris, France

\subsection{6/archdischild-2014-307384.344}

Background and aims There is concern that declines in mortality after very preterm birth are accompanied by higher morbidity as more fragile babies survive. We investigated whether rates of severe neonatal morbidity were higher in regions with low versus high mortality.

Methods The EPICE cohort included all births between $22+0$ and $31+6$ weeks of gestation in 19 European regions in 20112012 ( $\mathrm{N}=7878$ live births). We divided the cohort into tertiles based on regional in-hospital mortality rates excluding deaths due to congenital anomalies: low $(<12.0 \%)$, medium $(12.0$ to $14.9 \%)$ and high $(\geq 15.0 \%)$. Severe neonatal morbidity was defined as intraventricular haemorrhage grades III-IV, cystic periventricular leukomalacia, surgery/peritoneal drainage for necrotizing enterocolitis or retinopathy of prematurity (grades $\geq 3$ ). Bronchopulmonary dysplasia (BPD) was analysed separately. Multilevel logistic regressions were used to model regional-level effects.

Results 6770 infants were discharged home. 12.2\% had at least one severe morbidity (regional range: $3.8 \%$ to $24.4 \%$ ) and $15.3 \%$ had BPD (regional range: $6.7 \%$ to $34.9 \%$ ). Rates of severe morbidity were $11.7 \%, 12.1 \%$ and $12.9 \%$ in low, medium and high mortality regions, respectively $(\mathrm{p}=0.45)$. BPD was higher in low mortality regions $(18.7 \%, 16.7 \%$ and $10.2 \%$, respectively $(\mathrm{p}<0.001)$. The odds ratio for severe morbidity associated with birth in a low versus high mortality region was 0.83 (95\% CI: 0.55-1.25) and for BPD was 1.81 (95\% CI: 1.08-3.0).

Conclusion Rates of severe neonatal morbidity and BPD vary greatly between regions in Europe. BPD rates were higher in lower mortality regions, but this was not true for other morbidities.

\section{PS-048 TRENDS IN SNAPPE-II AND CRIB II SCORES OVER A 15-YEAR PERIOD}

MC de VoS, EJH Mulder, J Schuurmans, D Kwakkel, JB Derks, F Groenendaal. Perinatal Center, Wilhelmina Children's Hospital University Medical Center Utrecht, Utrecht, Netherlands

\subsection{6/archdischild-2014-307384.345}

Background During the last decades mortality of preterm born neonates has declined in the Western world.
Aim We hypothesised that the decrease in mortality in very preterm neonates over a period of 15 years was associated with a decrease in illness severity scores (SNAPPE-II and CRIB II).

Subjects/methods Inborn neonates (January 1997 until December $2011 ; \mathrm{n}=928$ ) with a gestational age of 26.0-29.9 weeks and without congenital malformations.

SNAPPE-II and CRIB II scores, mortality ( $<120$ days $)$ and morbidity (severe intraventricular haemorrhage (IVH), severe cystic periventricular leukomalacia (cPVL), bronchopulmonary dysplasia (BPD), perforated necrotizing enterocolitis (NEC) and severe retinopathy of prematurity (ROP)) were recorded. Based on important changes in care, subjects were categorised into three periods of year of birth (1: 1997-1999, 2: 2000-2005, and 3: 2006-2011) and outcomes were analysed using multivariable analysis including SNAPPE-II/CRIB II scores, period, gestational age, gender, and their interactions.

Results SNAPPE-II and CRIB II scores were significantly lower for gestational ages between 26.0 and 29.9 in period 3 versus period 1 ( $\mathrm{p}=0.002 ; \mathrm{p}=0.018$, respectively) in a cubic model analysis. The risks of mortality and serious morbidity were significantly lower for similar SNAPPE-II and CRIB II scores in period 3 versus period 1 ( $\mathrm{p}=0.010 ; \mathrm{p}=0.041$, respectively). Females had a significantly lower risk of mortality and serious morbidity than males ( $\mathrm{p}=0.031 ; \mathrm{p}<0.0001$, respectively).

Conclusions SNAPPE-II and CRIB II scores decreased over a 15 -year period. Meanwhile, the risk of mortality and serious morbidity for similar SNAPPE-II and CRIB II scores decreased suggesting substantial improvement in perinatal care.

\section{PS-049 QUALITY APPRAISAL OF INTERNATIONAL GUIDELINES ON PERINATAL CARE OF EXTREMELY PREMATURE INFANTS}

${ }^{1} \mathrm{~N}$ Binepal, ${ }^{2} \mathrm{G}$ Moore, ${ }^{2} \mathrm{~B}$ Lemyre, ${ }^{2} \mathrm{~T}$ Daboval, ${ }^{3} \mathrm{~S}$ Dunn, ${ }^{4} \mathrm{~S}$ Leduc. ${ }^{1}$ Pediatrics, Children's Hospital of Eastern Ontario, Ottawa, Canada; ${ }^{2}$ Neonatology, Children's Hospital of Eastern Ontario, Ottawa, Canada; ${ }^{3}$ Children's Hospital of Eastern Ontario Research Institute, Children's Hospital of Eastern Ontario, Ottawa, Canada; ${ }^{4}$ Life Sciences, University of Ottawa, Ottawa, Canada

\subsection{6/archdischild-2014-307384.346}

Background Clinicians often refer to published or local guidelines when counselling expectant parents on perinatal care decisions at the limits of viability. The number and quality of guidelines used worldwide is unknown.

Objectives Assess quality of international guidelines regarding perinatal care of 22-25 wk GA infants.

Methods MEDLINE, Pre-MEDLINE and TRIP databases were searched for international guidelines using specific criteria. Titles/abstracts were screened and the final selected guidelines were reviewed and appraised by two reviewers using the validated AGREE-II (Appraisal of Guidelines for Research and Evaluation Instrument) tool. This instrument assesses guideline quality (scope, stakeholder involvement, rigour/transparency, clarity, applicability, editorial independence). Items with score differences $>3$ were reviewed for discussion.

Results Database and grey search yielded 263 publications. Screening left 37 guidelines, 16 of which met all inclusion criteria. Regions represented included North America, Australia and Europe. Appraisal using the AGREE-II tool by three independent appraisers revealed deficits within all domains, predominantly 'rigour of development' and 'applicability'. Overall quality scores 
ranged from 11\%-61\%; no guideline was deemed suitable for use. Only Swiss and Canadian guidelines were considered useable with significant modifications.

Conclusion Several international guidelines on perinatal care of 22-25 wk GA infants exist. Using the AGREE-II tool, we identified many deficits in the quality of these guidelines. Not a single guideline was deemed suitable for use using the AGREE-II tool. Use of poorly developed guidelines may be detrimental to decision-making, thus there is a need for transparent and rigorous guidelines regarding the perinatal care of 22-25 wk GA infants.

\section{PS-050 QUALITY OF LIFE FOR PARENTS OF VERY-LOW BIRTH WEIGHT INFANTS ENROLLED IN A CLINICAL STUDY}

${ }^{1} \mathrm{~T}$ Nordheim, ${ }^{2} \mathrm{~T}$ Rustøen, ${ }^{1} \mathrm{~B}$ Nakstad. 'Department of Children and Adolescents Medicine, Akershus University Hospital, Lørenskog, Norway; ${ }^{2}$ Acute Clinic, Oslo University Hospital, Oslo, Norway

10.1136/archdischild-2014-307384.347

Background In this study we wanted to evaluate if parents of very-low birth weight (VLBW) infants enrolled in a clinical study developed a lower quality of life compared to parents from a control sample.

Methods We recruited parents of children attending the Norwegian multicenter study for premature nutrition (PreNu). The PreNu study was a randomised clinical nutritional trial, where 50 VLBW-children $(<1500 \mathrm{~g})$ were recruited within the first hours of their life. We also recruited parents of VLBW-children born immediately before and after the recruitment period of the PreNu study, to serve as a control group.

The parents $(n=63)$ were given a questionnaire with validated measures on quality of life (Quality of Life Scale), anxiety and depression (Hospital Anxiety and Depression Scale), fatigue (Lee Fatigue Scale), sleeping disturbance (General Sleep Disturbance Scale), pain (Brief Pain Inventory), comorbidity (SelfAdministered Comorbidity Questionnaire) and hope (Herth Hope Index). The parents were asked to answer as they would have done at the time their children were at the NICU.

Results The response rate was 69\%. T-tests showed no significant difference between the groups on all measures except for quality of life. The PreNu parents rated their quality of life significantly higher than the control group ( $p=0.018$ ).

Conclusion Our fear that the parents of the PreNu-children suffered an intolerable burden seems unfounded. The results suggest that being the parent of a VLBW-child attending a clinical study is not a burden, but may in fact be an enrichment.

\section{PS-051 RANDOMISED CONTROLLED TRIALS IN VERY PRETERM INFANTS: DOES INCLUSION IN THE STUDY RESULT IN ANY LONG-TERM BENEFIT?}

${ }^{1} \mathrm{CM}$ Rüegger, ${ }^{2} \mathrm{~A}$ Kraus, ${ }^{1} \mathrm{~B}$ Koller, ${ }^{3} \mathrm{G}$ Natalucci, ${ }^{3} \mathrm{~B}$ Latal, ${ }^{1} \mathrm{E}$ Waldesbühl, ${ }^{1} \mathrm{JC}$ Fauchère, ${ }^{2} \mathrm{~L}$ Held, ${ }^{1} \mathrm{HU}$ Bucher. 'Division of Neonatology, University Hospital Zurich, Zurich, Switzerland; ${ }^{2}$ Division of Biostatistics, University of Zurich, Zurich, Switzerland; ${ }^{3}$ Child Development Center, University Children's Hospital, Zurich, Switzerland

\subsection{6/archdischild-2014-307384.348}

Background Since the introduction of randomised controlled trials (RCT) in clinical research, there has been discussion of whether enrolled patients have worse or better outcomes than comparable nonparticipants.
Objective To investigate whether very preterm infants randomised to a placebo group in a RCT have equivalent neurodevelopmental outcomes to infants who were eligible but not randomised (eligible NR).

Methods In the course of an RCT investigating the neuroprotective effect of early high dose erythropoietin on the neurodevelopment of very preterm infants, the outcome data of 72 infants randomised to placebo were compared with those of 108 eligible NR infants. Our primary outcome measures were the mental (MDI) and psychomotor (PDI) developmental indices of the Bayley Scales of Infant Development II at 24 months corrected age. The outcomes of the two groups were considered equivalent if the confidence intervals of their mean differences fitted within our \pm 5 point margin of equivalence.

Results Except for a higher socioeconomic status of the trial participants, both groups were balanced for most perinatal variables. The mean difference $(90 \% \mathrm{CI})$ between the placebo and the eligible NR group was -2.1 (-6.1 and 1.9) points for the MDI and -0.8 ( -4.2 and 2.5 ) points for the PDI (in favour of the placebo group). After adjusting for the socioeconomic status, maternal age and child age at follow-up, the mean difference for the MDI was -0.5 (-4.3 and 3.4) points.

Conclusions Our results indicate that the participation of very preterm infants in an RCT is associated with equivalent longterm outcomes compared to non-participating infants.

\section{PS-052 SETTING PRETERM BIRTH RESEARCH PRIORITIES WITH MULTIPLE PROFESSIONS AND SERVICE USERS IN THE UK}

${ }^{1} S$ Uhm, ${ }^{2} \mathrm{~F}$ Alderdice, ${ }^{3} \mathrm{I}$ Brady, ${ }^{1} \mathrm{~B}$ Chambers, ${ }^{4} \mathrm{Z}$ Chivers, ${ }^{5} \mathrm{~S}$ Crowe, ${ }^{6} \mathrm{AL}$ David, ${ }^{7} \mathrm{~S}$ Deshpande, ${ }^{8} \mathrm{C}$ Gale, ${ }^{9} \mathrm{G}$ Gyte, ${ }^{6} \mathrm{CP}$ James, ${ }^{10} \mathrm{~L}$ Duley, ${ }^{11} \mathrm{~J}$ McNeill, ${ }^{12} \mathrm{~A}$ Shennan, ${ }^{13} \mathrm{MA}$ Turner, ${ }^{1} \mathrm{~S}$ Oliver. ${ }^{1}$ Institute of Education, University of London, London, UK; ${ }^{2}$ The Premature Baby Charity for Northern Ireland, TinyLife, Carryduff, UK; ${ }^{3}$ rrish Premature Baby, Irish Premature Baby, Dublin, Ireland; ${ }^{4}$ The Premature and Special Care Baby Charity, Bliss, London, UK; ${ }^{5}$ James Lind Alliance, James Lind Alliance, London, UK; ${ }^{6}$ University College London, Institute for Women's Health, London, UK; ' British Association of Perinatal Medicine, British Association of Perinatal Medicine, London, UK; ${ }^{8}$ Academic Neonatal Medicine, Imperial College, London, UK; ${ }^{9}$ National Childbirth Trust, National Childbirth Trust, London, UK; ${ }^{10}$ University of Nottingham, Nottingham Clinical Trials Unit, Nottingham, UK; ${ }^{11}$ The Premature Baby Charity for Northern Ireland, TinyLife, Carryduf, UK; ${ }^{12}$ Kings College London, Kings College London, London, UK; ${ }^{13}$ Liverpool Women's NHS Foundation Trust, Liverpool Women's NHS Foundation Trust, Liverpool, UK

\subsection{6/archdischild-2014-307384.349}

Background Preterm birth is the most important determinant of adverse infant outcomes. Research agendas in this area have been determined primarily by researchers.

Objectives To identify and prioritise future research areas in preterm birth that are most important to service users and practitioners.

Methods A priority setting partnership was established with families with experience of preterm birth and organisations representing them, obstetricians, neonatologists, midwives and neonatal nurses. Research uncertainties were gathered from surveys and analysis of systematic reviews and clinical guidance. Prioritisation was through voting; final ranking occurred at a facilitated workshop, as advocated by the James Lind Alliance.

Results 593 uncertainties were submitted by 386 respondents (58\% service users, 30\% healthcare professionals and 12\% from those in both roles); 52 were identified from literature 